\title{
Snow dielectric properties: from DC to microwave X-band
}

\author{
Th. AChammer and A. Denoth \\ Institute of Experimental Physics, University of Innsbruck, A-6020, Austria
}

\begin{abstract}
Broadband measurements of dielectric properties of natural snow samples near or at $0^{\circ} \mathrm{C}$ are reported. Measurement quantities are: dielectric permittivity, loss factor and complex propagation factor for electromagnetic waves. $\mathrm{X}$-band measurements were made in a cold room in the laboratory; measurements at low and intermediate frequencies were carried out both in the field (Stubai Alps, $3300 \mathrm{~m}$; Hafelekar near Innsbruck, $2100 \mathrm{~m}$ ) and in the cold room. Results show that in the different frequency ranges the relative effect on snow dielectric properties of the parameters: density, grain-size and shape, liquid water content, shape and distribution of liquid inclusions and content of impurities, varies significantly. In the low-frequency range the influence of grain-size and shape and snow density dominates; in the medium-frequency range liquid water content and density are the dominant parameters. In the microwave X-band the influence of the amount, shape and distribution of liquid inclusions and snow density is more important than that of the remaining parameters.
\end{abstract}

\section{INTRODUCTION}

Measurements of the spectral behaviour of the dielectrical properties, especially in the X-band, have been reported by several authors, with somewhat inconsistent results. The peak of the losses has been found not only at $\approx 10 \mathrm{GHz}$ (Hallikainen and others, 1982; Mätzler, 1987) but also at frequencies higher than $12 \mathrm{GHz}$ (Linlor, 1980). In addition, experimental evidence of magnetic effects $\left(\mu^{\prime} \leq 1\right.$ and $\left.\mu^{\prime \prime} \geq 0\right)$ induced by ring-shaped water inclusions has been reported (Mätzler, 1988). The aim of this work was to study the relative influence of such different parameters as porosity, density, liquid content and shape, grain-size and shape on the dielectric behaviour of natural snow samples over a wide frequency range. By measuring the complex reflection and complex transmission coefficient in the microwave X-band, dielectric and magnetic properties have been derived separately.

\section{MEASUREMENT TEGHNIQUES}

Different measurement techniques have been used to measure the dielectric permittivity of snow in the different frequency ranges. In the low-frequency range from $10 \mathrm{~Hz}$ up to $50 \mathrm{MHz}$ measurements were made with a simple plate condenser (consisting of 5 or 8 plates; dimension $6 \times 7 \mathrm{~cm}^{2}$ with a spacing of $1.5 \mathrm{~cm}$ ) as sample holder and a low-frequency automatic network analyzer (ANA), type R\&S ZPV with tuner unit El (Rohde and Schwarz, 1978). The frequency dependence of the sensor impedance in snow is automatically and continuously recorded. A block diagram of the experimental setup is given in Figure la. From the complex ratio of the
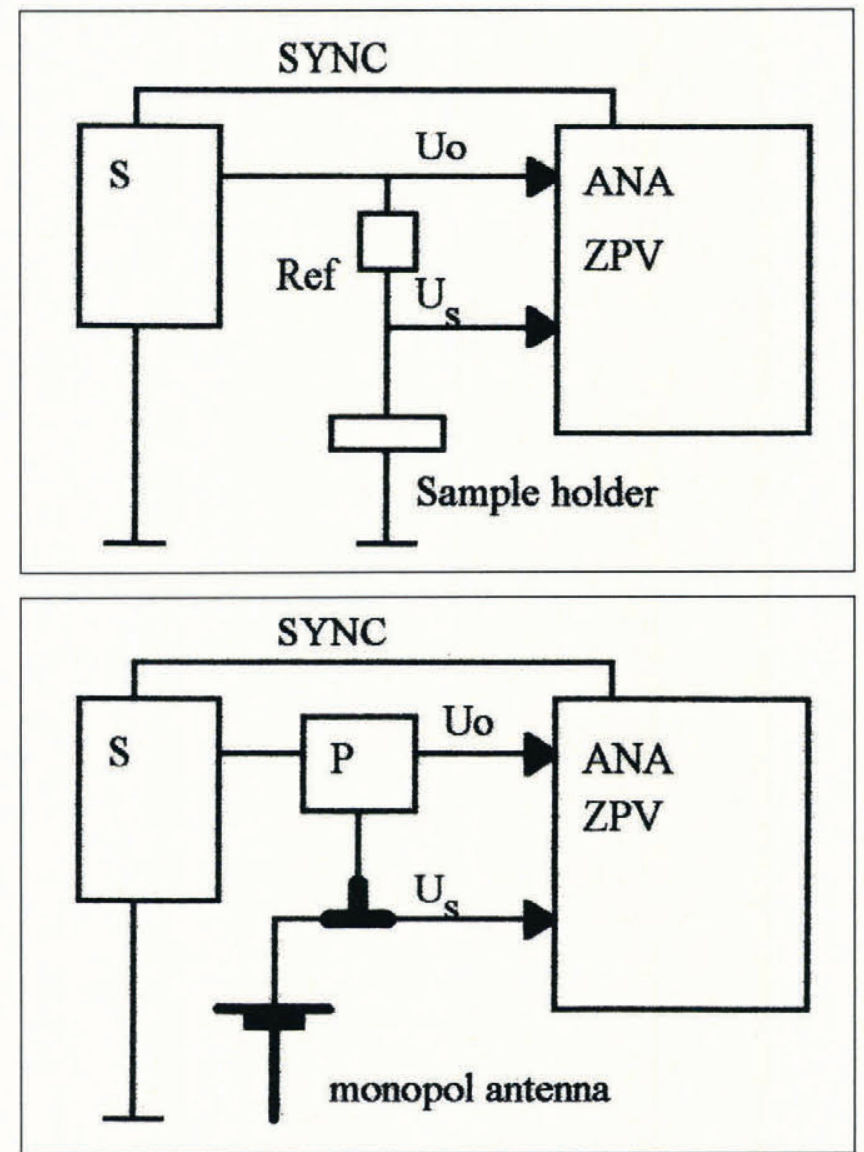

Fig. 1. Block diagram of the measuring systems. a, lowfrequency. $S$, sweeper; $A \mathcal{N} A$, automatic network analyzer ZPV; SYNC, synchronisation; $U_{\mathrm{o}}, U_{\mathrm{s}}$, measured signals; Ref, reference impedance. $b$, intermediate frequency. $S$, sweeper; $P$, power splitter; $A \mathcal{N} A$, automatic network analyzer $Z P V ; U_{\mathrm{o}}, U_{\mathrm{s}}$, measured signals. 
measurement voltages, $U_{\mathrm{o}}$ and $U_{\mathrm{s}}$, the dielectric function can be calculated.

In the intermediate frequency range from $50 \mathrm{MHz}$ to approximately $1.5 \mathrm{GHz}$ measurements were made with a set of monopol antennas as resonators (Denoth and Foglar, 1986) together with the network analyzer ZPV and tuner unit E3, whereby a specific measuring system, denoted as T-method, was applied (Rohde and Schwarz, 1978). From the measured change of sensor resonance frequency between that in air and with the sensor in snow, and from the measured change of half width of the resonance curve, the dielectric function was calculated. A block diagram of the experimental setup is given in Figure $1 \mathrm{~b}$.

In the microwave X-band a "free space measurement technique" was applied by using a high-frequency network analyzer (type HP8510A). This method was preferred, because it allows measurements on large snow samples with dimension $50 \times 50 \mathrm{~cm}^{2}$ cross section and different thicknesses to average over possible snow inhomogeneities. A block diagram of the X-band measurement setup is given in Figure 2. From the measured complex reflection coefficient $R$ and the

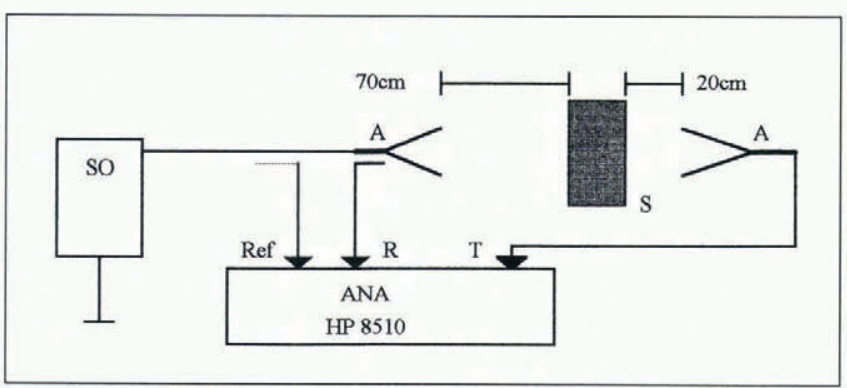

Fig. 2. Block diagram of the X-band measuring system. SO, sweeper oscillator; ANA, automatic network analyzer; $S$, sample; $A$, horn antennas; Ref, reference signal; $R$, reflected signal; $T$, transmitted signal.

complex transmission coefficient $T$, which includes effects of multiple beam interference (Azzam and Bashara, 1977), the dielectric function of snow was derived.

For interpretation of snow dielectric function, such texture parameters as snow density $\rho$, grain-size and shape, liquid water content $W$ and liquid shape have to be known. Snow density was measured by weighing a known volume of a snow sample. Grain-size and shape were derived from an analysis of snow photographs (Denoth, 1982). Liquid water content $W$ was measured using a freezing calorimeter, whereby the relative measuring error was found to be approximately $0.7 \mathrm{Vol} \%$ for measurements in the laboratory and $\approx 1 \mathrm{Vol} . \%$ for field measurements. In addition to these measurements snow liquid water content was also measured using a calibrated dielectric sensor, whereby the relative error was found to be $\approx 0.2 \mathrm{Vol} . \%$.

\section{EXPERIMENTAL SNOW DIELECTRIC DATA}

Measurements were made with different snow samples, which were classified according to grain-size and shape.
Snow samples with well rounded grains, grain-size $>0.5 \mathrm{~mm}$, were denoted as old coarse-grained snow. Fine-grained snow samples (grain-size $<0.5 \mathrm{~mm}$ ) with a

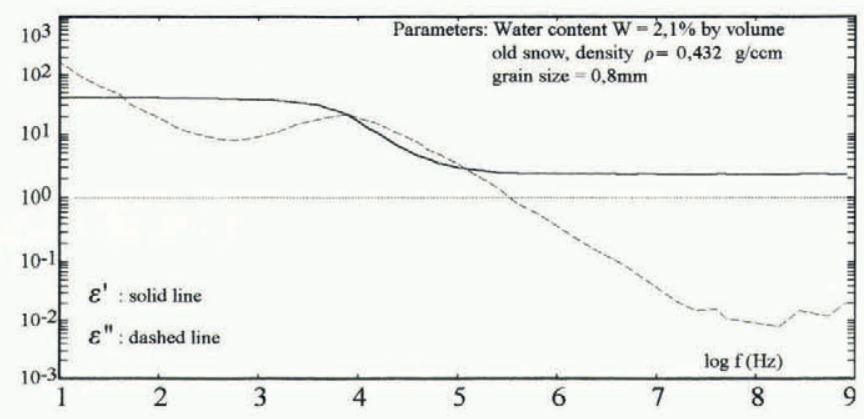

Fig. 3. Dielectric function of snow in the low and intermediate frequency regime.

plate-like and/or needle-like shape of the grains were denoted as new fine-grained snow.

The principle behaviour of permittivity and the loss factor of snow in the frequency regime from $10 \mathrm{~Hz}$ to $1 \mathrm{GHz}$ is shown in Figure 3. Due to the relaxation process of the ice component near $10 \mathrm{kHz}$, permittivity decreases from its static value to a relatively low but frequencyindependent value in the range of $1 \mathrm{MHz}$ to approximately $1 \mathrm{GHz}$. The low-frequency losses are determined by ionic conductivity and by the dielectric loss of the ice

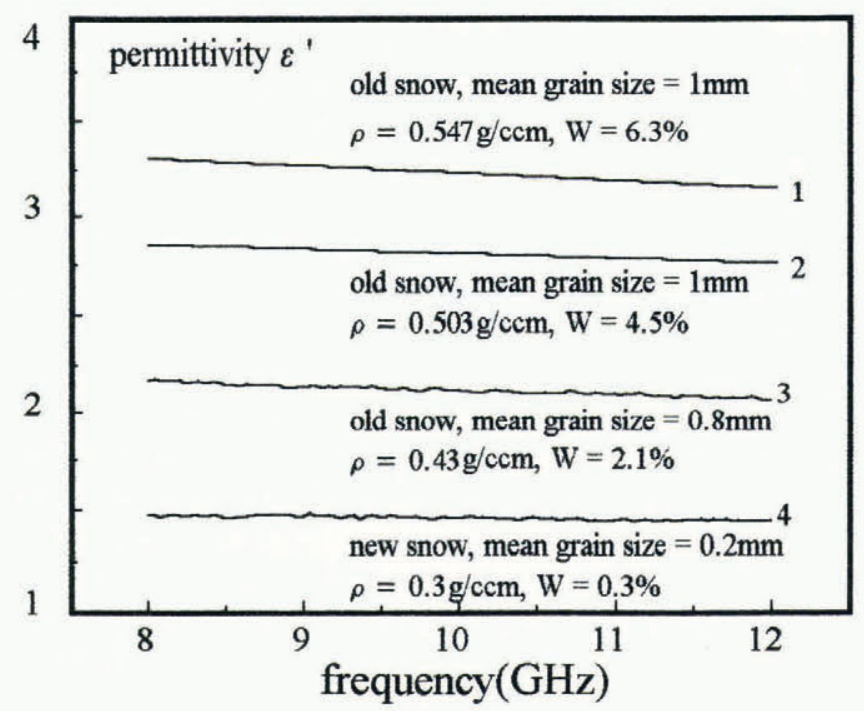

Fig. 4. Permittivity $\epsilon^{\prime}$ of snow between 8 and $12 \mathrm{GHz}$.

component. In the $\mathrm{MHz}$ regime the losses are very low even if the snow is wet. In the microwave X-band measurements were made with moderately wet natural snow samples. The dielectric function, $\epsilon^{\prime}$ and $\epsilon^{\prime \prime}$, of four selected snow samples is shown in Figures 4 and 5. In general, snow permittivity shows a slight decrease with increasing frequency, the loss factor shows a slight increase with increasing frequency. Except in sample 4 (new fine-grained snow, $\rho=0.3 \mathrm{~g} \mathrm{~cm}^{-3}, W=0.3 \%$ ) a 


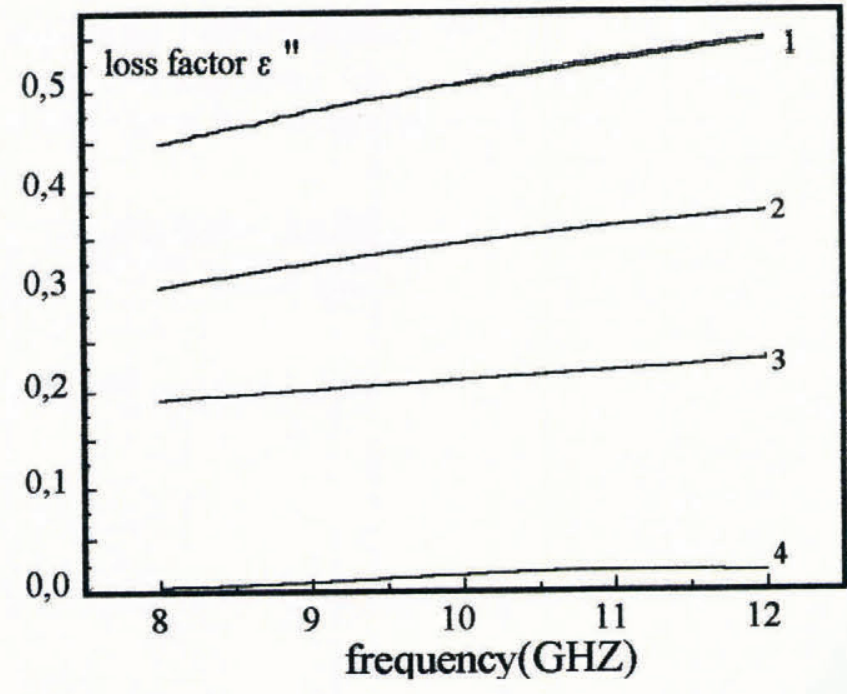

Fig. 5. Loss factor $\epsilon^{\prime \prime}$ of snow between 8 and $12 \mathrm{GHz}$.

maximum of the losses in the microwave X-band was not observed. In addition, no significant deviation from $\mu^{\prime}=1$ and $\mu^{\prime \prime}=0$, as a possible magnetic effect of ringshaped water inclusions, was detected.

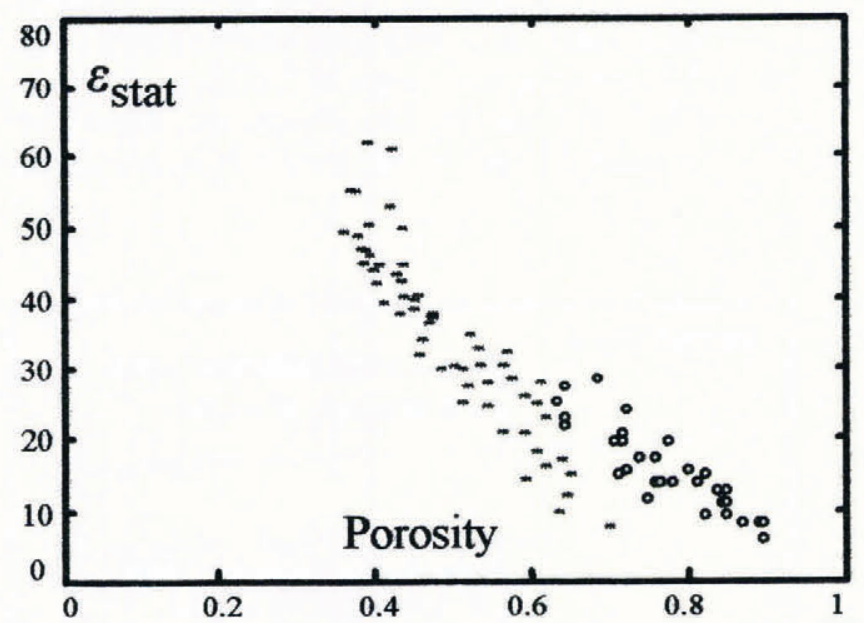

Fig. 6. Dependence of static dielectric constant on porosity. (Open symbols, new fine-grained snow; asterisks, old coarse-grained snow.)

\section{RESULTS AND CONCLUSIONS}

Snow dielectric data in different frequency regimes have been analyzed with respect to the effects of porosity, density, water content and type of snow (grain-size and shape). Results of measurements are given for three selected frequencies: $<1 \mathrm{kHz}$ (static values), $10 \mathrm{MHz}$ and $10 \mathrm{GHz}$, respectively. Figure 6 shows the dependence of the static dielectric constant on snow porosity, whereby the type of snow plays an important role. Figure 7 shows the influence of the liquid water content. Due to the similar static permittivity of ice and water only a weak influence of liquid water content was observed.

Figure 8 shows the dependence of the incremental permittivity $\Delta \epsilon^{\prime}$ on water content at a measurement

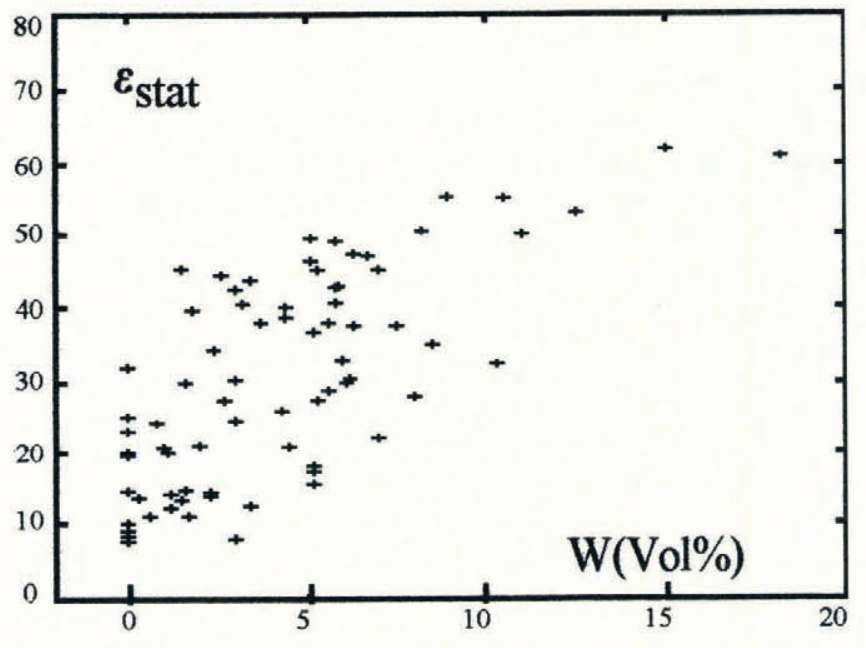

Fig. 7. Dependence of static dielectric constant on water content.

frequency of $10 \mathrm{MHz}$, whereby $\Delta \epsilon^{\prime}$ is defined as:

$$
\Delta \epsilon^{\prime}=\epsilon_{\text {measured }}-1-1.76(1-\Phi)-0.37(1-\Phi)^{2}
$$

$\Delta \epsilon^{\prime}$ depends on water content but no significant effect of grain-size or shape was observed. Figure 9 shows the dependence of the loss factor $\epsilon^{\prime \prime}$ on snow porosity. $\epsilon^{\prime \prime}$ shows a strong dependence on both snow porosity and snow type; a significant influence of water content, however, could not be found. The comparable higher losses of new fine-grained snow may be due to the relatively larger surface of the ice grains, surface conductivity possibly increasing in importance.

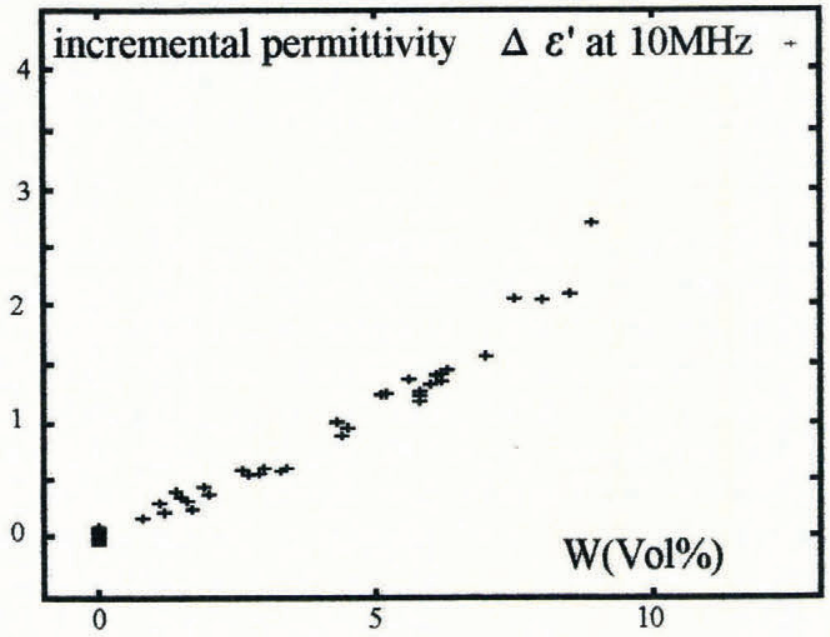

Fig. 8. Incremental permittivity $\Delta \epsilon^{\prime}$ for new fine-grained and old coarse-grained snow.

Results of X-band measurements are shown in Figures 10 and 11. Figure 10 shows the dependence of the incremental permittivity $\Delta \epsilon^{\prime}(10 \mathrm{GHz})$ on water content. Figure 11 gives the dependence of $\epsilon^{\prime \prime}$ on liquid content for new fine-grained and old coarse-grained snow samples.

No significant effect of the type of snow on $\Delta \epsilon^{\prime}$ $(10 \mathrm{GHz})$ was found. Water content is the dominant parameter; calculations of $\Delta \epsilon^{\prime}$ based on the Polder and 


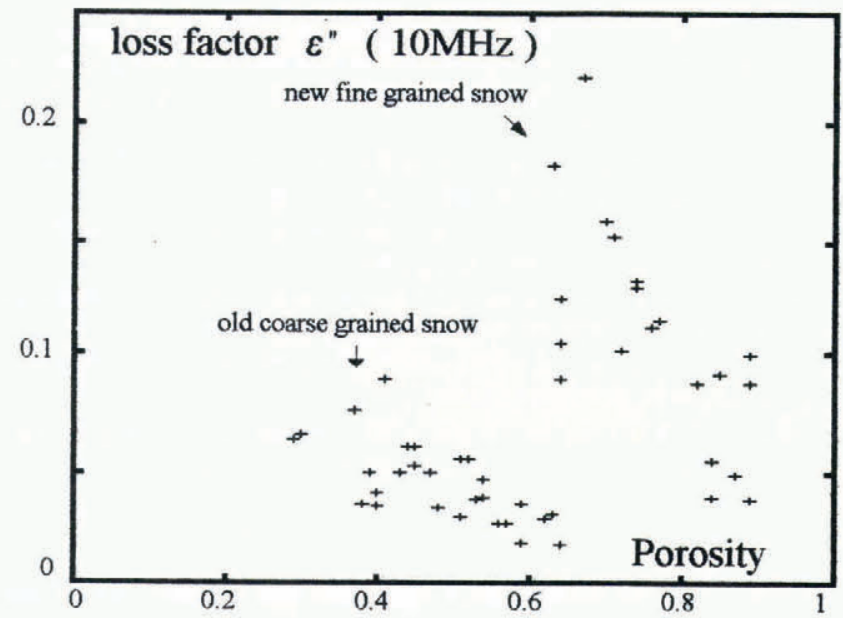

Fig. 9. Loss factor $\epsilon^{\prime \prime}$ for new fine-grained and old coarsegrained snow.

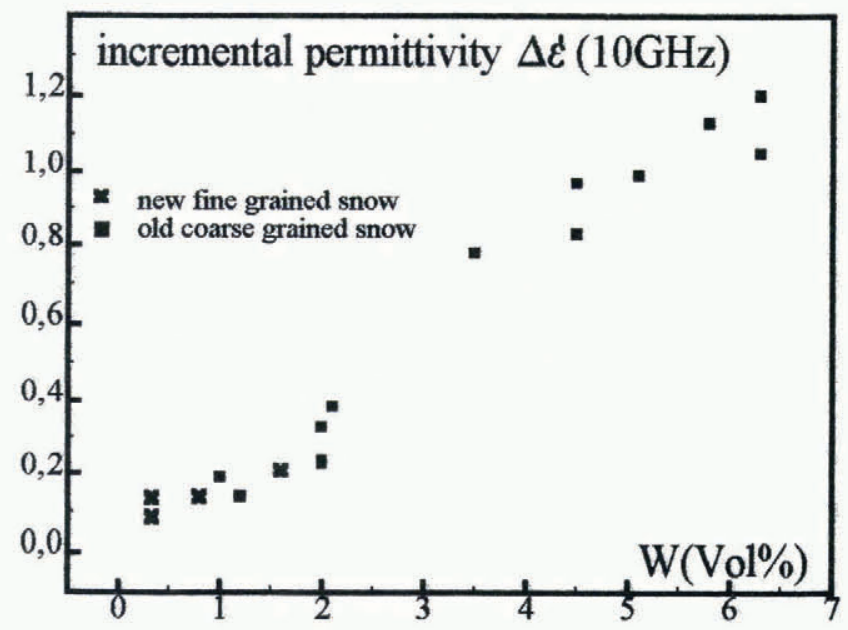

Fig. 10. Incremental permittivity $\Delta \epsilon^{\prime}$ for new fine-grained and old coarse-grained snow.

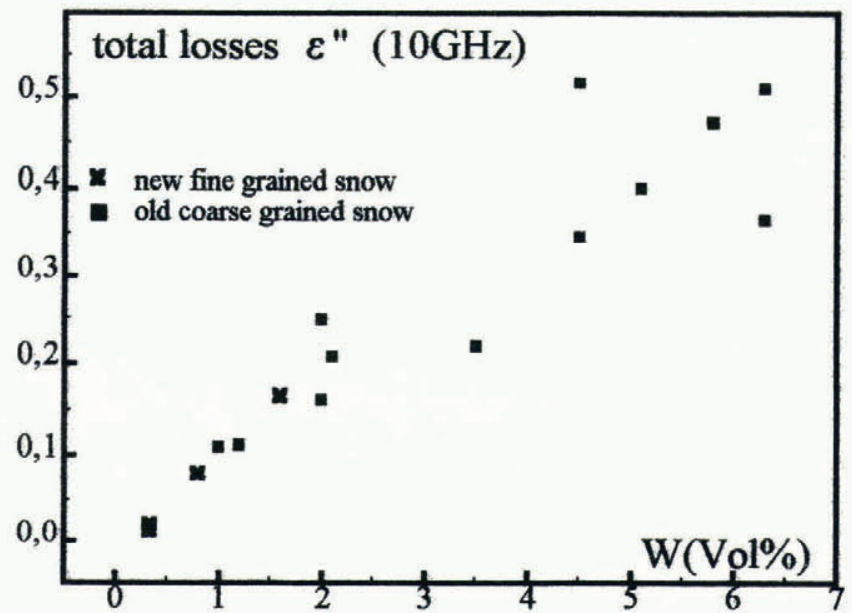

Fig. 11. Loss factor $\epsilon^{\prime \prime}$ for new fine-grained and old coarse-grained snow.

van Santen (1946) theory indicate a weak influence of the shape of the water component. The loss factor $\epsilon^{\prime \prime}$ $(10 \mathrm{GHz})$ shows a strong dependence on water content. Again, model calculations indicate an influence of the shape of water component. This theory predicts, however, a considerable shift in the apparent relaxation frequency which has not always been observed. So, scattering as an additional effect should be considered and modelling of $\epsilon^{\prime \prime}$ data based solely on water content and shape factors seems not to be sufficient.

The relative importance of the parameters porosity, liquid content, liquid shape, grain-size and shape in the different frequency regimes is summarized in Table 1 .

\section{ACKNOWLEDGEMENTS}

The Fonds zur Förderung der Wissenschaftlichen Forschung is thanked for supporting in part these

Table 1. Relative importance of the parameters porosity, liquid content, liquid shape, grain-size and shape for the dielectric function of snow

$\epsilon^{\prime} \quad \epsilon^{\prime \prime}$

Important

Less important

Not important

Important

Less important

Not important

Important

Less important

Not important low-frequency regime: $<1 \mathrm{kHz}$ grain-size and shape; porosity liquid content impurities (DC-conductivity) liquid shape

$10 \mathrm{MHz}$

liquid content; porosity

liquid shape

grain-size and shape

grain-size and shape; porosity impurities?

liquid content

$10 \mathrm{GHz}$

liquid content; porosity grain shape; liquid shape liquid content and shape scattering? impurities? porosity 
measurements through project P5907. The Wintersport Tirol AG is thanked for supporting in part the field activities in the Stubai Alps. The authors thank the Österreichischen Forschungsgemeinschaft for supporting this work through grant numbers 06/2204 and 06/2205.

\section{REFERENCES}

Azzam, R. M. A. and N. M. Bashara. 1977. Ellipsometry and polarized light. Amsterdam, etc., North-Holland Publishing Company.

Denoth, A. 1982. Effect of grain geometry on electrical properties of snow at frequencies up to $100 \mathrm{MHz}$. J. Appl. Phys., 53(11), 74967501.

Denoth, A. and A. Foglar. 1986. Recent developments of snow moisture dielectric devices. In Proceedings of the International Snow Science Workshop, Lake Tahoe, CA, 22-25 October 1986, 72-76.
Hallikainen, M., F. T. Ulaby and N. Abdelrazik. 1982. Measurements of the dielectric properties of snow in $4-18 \mathrm{GHz}$ frequency range. In 12th European Microwave Conference, Helsinki, Finland, 13-17 September 1982. Proceedings, 151-156.

Linlor, W. I. 1980. Permittivity and attenuation of wet snow between 4 and $12 \mathrm{GHz}$. 7. Appl. Phys., 51(5), 2811-2816.

Mätzler, C. 1987. Applications of the interaction of microwaves with the natural snow cover. Remote Sensing Rev., 2(2), 256-259.

Mätzler, C. 1988. Eddy currents in heterogeneous mixtures. Journal of Electromagnetic Waves and Applications, 2(5/6), 473-479.

Polder, D. and J.H. van Santen. 1946. The effective permeability of mixtures of solids. Physica, $\mathbf{8}(5)$.

Rohde, L. and H. Schwarz, eds. 1978. Impedanztechnik. Rohde and Schwarz GmbH. (Technical notes 83.)

The accuracy of references in the text and in this list is the responsibility of the authors, to whom queries should be addressed. 